\title{
Blue Light Perception by Both Roots and Rhizobia Inhibits Nodule Formation in Lotus japonicus
}

\author{
Aya Shimomura, ${ }^{1,2}$ Ayumi Naka, ${ }^{2}$ Nobuyuki Miyazaki, ${ }^{2}$ Sayaka Moriuchi, ${ }^{2}$ Susumu Arima, ${ }^{1,2}$ \\ Shusei Sato, ${ }^{3}$ Hideki Hirakawa, ${ }^{4}$ Makoto Hayashi, ${ }^{5}$ Maskit Maymon, ${ }^{6}$ Ann M. Hirsch, ${ }^{6,7}$ and \\ Akihiro Suzuki ${ }^{1,2}$ \\ ${ }^{1}$ United Graduate School of Agricultural Sciences, Kagoshima University, Korimoto, Kagoshima 890-0065, Japan; ${ }^{2}$ Department \\ of Agro-Environmental Sciences, Faculty of Agriculture, Saga University, Honjyo-machi, Saga, Saga 840-8502, Japan; \\ ${ }^{3}$ Department of Environmental Life Sciences, Tohoku University, Katahira, Aoba-ku, Sendai 980-8577, Japan; ${ }^{4}$ Kazusa DNA \\ Research Institute, Kazusa-kamatari, Kisarazu, Chiba 292-0818, Japan; ${ }^{5}$ RIKEN Plant Science Center, Yokohama, Kanagawa \\ 230-0045, Japan; ${ }^{6}$ Department of Molecular, Cell and Developmental Biology, University of California-Los Angeles, Los \\ Angeles, CA 90095-1606, U.S.A.; and ${ }^{7}$ Molecular Biology Institute, University of California-Los Angeles
}

Accepted 6 September 2016.

\begin{abstract}
In many legumes, roots that are exposed to light do not form nodules. Here, we report that blue light inhibits nodulation in Lotus japonicus roots inoculated with Mesorhizobium loti. Using RNA interference, we suppressed the expression of the phototropin and cryptochrome genes in L. japonicus hairy roots. Under blue light, plants transformed with an empty vector did not develop nodules, whereas plants exhibiting suppressed expression of cry 1 and cry 2 genes formed nodules. We also measured rhizobial growth to investigate whether the inhibition of nodulation could be caused by a reduced population of rhizobia in response to light. Although red light had no effect on rhizobial growth, blue light had a strong inhibitory effect. Rhizobial growth under blue light was partially restored in signature-tagged mutagenesis (STM) strains in which LOV-HK/PAS- and photolyaserelated genes were disrupted. Moreover, when Ljcry1A and Ljcry $2 B$-silenced plants were inoculated with the STM strains, nodulation was additively increased. Our data show that blue light receptors in both the host plant and the symbiont have a profound effect on nodule development. The exact mechanism by which these photomorphogenetic responses function in the symbiosis needs further study, but they are clearly involved in optimizing legume nodulation.
\end{abstract}

Plants rely on environmental cues for survival. Light is one such cue and plants perceive its quality, intensity, and direction. Phototropins (PHOT) and cryptochromes (CRY) are wellknown blue light and UV-A photoreceptors and phytochromes (PHY) are red and far-red light receptors (Christie et al. 1999 2015; Gressel 1979; Mockler et al. 2003). PHOT, which bind the blue light-absorbing chromophore FMN, harbor two FMNbinding domains, LOV1 and LOV2 (light, oxygen, voltage1,

Nucleotide sequence data for Atphot1, Atphot2, Atcry1, Atcry2, and Atcry3 are available from the DDBJ database under accession numbers AF360218, AF053941, AY124863, BT008576, and AY102138, respectively.

Corresponding author: A. Suzuki; Telepehone/Fax: +81-952-28-8721; E-mail: azuki@cc.saga-u.ac.jp

*The $\boldsymbol{e}$-Xtra logo stands for "electronic extra" and indicates that twelve supplementary figures and one supplementary table are published online.

(C) 2016 The American Phytopathological Society and voltage 2 ) at the $\mathrm{N}$-terminus, and a serine/threonine kinase domain at the C-terminus (Briggs et al. 2001; Christie et al. 1998; Huala et al. 1997). LOV domains belong to the PerARNT-Sim (PAS) family (Christie et al. 1999). PHOT control phototropism in seedlings, induce stomatal opening, and regulate chloroplast movement (Han et al. 2013; Zhao et al. 2013). CRY have two recognizable domains, a DNA photolyase-like domain at the N-terminus and C-terminal DQXVP acidic-STAES domains that are distinguished mainly by their C-terminal extensions (Ahmad and Cashmore 1993; Li et al. 2013; Sakai and Haga 2012). CRY participate in the circadian clock, anthocyanin biosynthesis, anthogenesis, and plant growth (Li et al. 2013; Sharma et al. 2014). It is well-known that light, which is perceived by photoreceptors, affects plant phenotypes by influencing phytohormones. For example, blue light perceived by PHOT induces phototropism via auxin translocation (Haga et al. 2005; Kanegae et al. 2000), whereas light-induced germination by PHY and CRY perception of light is induced by gibberellic acid (Oh et al. 2009).

Leguminous plants and rhizobia establish a symbiosis in which root nodules develop on a host root. Within the nodules, rhizobia fix atmospheric nitrogen into ammonia, which eventually results in the synthesis of amino acids that are utilized by the host. In return, the plants provide photosynthetic products to the rhizobia as an energy source that drives the nitrogenfixation process (Bethlenfalvay and Phillips 1977; Sasaki et al. 2014; Zuanazzi et al. 1998). Light perceived by the aboveground parts of the plant is essential for the establishment of this symbiosis. Previously, we reported that not only light quantity but, also, light quality affects nodulation and, moreover, that this photomorphogenetic event is controlled by phytochrome through jasmonic acid signaling in Lotus japonicus (Suzuki et al. 2011). Recently, Weller et al. (2015) reported that ethylene signaling influences phytochrome regulation in pea, and ethyleneinsensitive mutants are known to have increased nodule numbers (Penmetsa and Cook 1997; Sun et al. 2006). Light triggers the suppression of nodulation in many legume roots. For example, previous studies reported that nodulation in Pisum sativum was decreased by root exposure to daylight (Rudin 1956) and that nodulation of isolated roots of Phaseolus vulgaris was suppressed by white light (Grobbelaar et al. 1971).

Like higher plants, many bacteria synthesize photoreceptors such as phytochrome (Auldridge and Forest 2011), and the analysis of numerous bacterial genomes has shown that photoreceptor proteins are present in many prokaryotes (Purcell and Crosson 
2008; van der Horst et al. 2007). For example, Giraud et al. (2002) reported that the Bradyrhizobium sp. strain ORS 278 genome has genes encoding phytochromes and Thompson and Sancar (2002) noted that Mesorhizobium loti synthesizes a photolyase that participates in blue light perception. Ogura et al. (2008a and b) described PAS/LOV proteins in Arabidopsis that have a PAS domain at the $\mathrm{N}$ terminus and a LOV domain at the $\mathrm{C}$ terminus, and Bonomi et al. (2012) found a LOV-HK/PAS protein (LPP) in Rhizobium leguminosarum bv. viceae 3841 .

In this study, we investigated the effects of light on the roots of Lotus japonicus Miyakojima MG20 and on the rhizobial strain (M. loti MAFF303099) to clarify the mechanisms of light perception required for nodulation in this symbiosis.

\section{RESULTS}

Nodulation is inhibited by light.

To study the effect of root exposure to light on nodulation in L. japonicus Miyakojima MG20, we employed three different strategies (Supplementary Fig. S1). Ten-day-old plants growing on agar plates were inoculated with M. loti MAFF303099 and the roots of some plants were shaded. Under unshaded conditions, both the shoot and root were exposed to continuous white light whereas, when the root was shaded, only the shoot was exposed. Under these conditions, shaded roots received approximately $10 \mu \mathrm{mol} \mathrm{m} \mathrm{m}^{-2} \mathrm{~s}^{-1}$ of light. Although root lengths were not significantly different between unshaded and shaded plants 21 days after inoculation (dai), the shoots from the unshaded plants were significantly shorter than those of the shaded plants (Fig. 1A and B). Also, unshaded roots had significantly fewer root nodules per plant than shaded roots (Fig. 1C), confirming earlier investigations that showed that nodulation is inhibited by white light. However, uninoculated plants did not differ in shoot length whether they were shaded or not (Supplementary Fig. S2), suggesting that the difference in shoot length of the inoculated plants grown under unshaded conditions is related to the presence of rhizobia.

In a split-root system in which the two root systems were inoculated with $M$. loti but one side of the root system was either totally shaded or both shaded and unshaded, we found
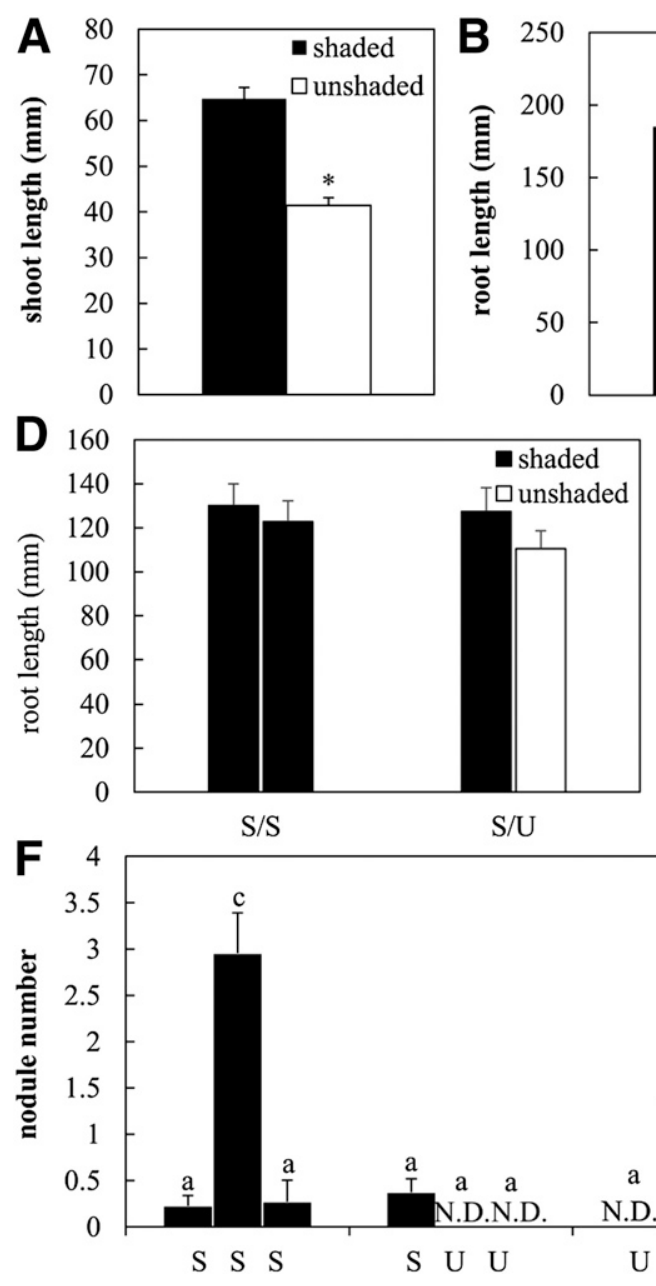

Fig. 1. Effect of white light on growth and nodulation in Lotus japonicus Miyakojima MG20 inoculated with Mesorhizobium loti MAFF303099. A to C, Plants were grown under continuous light $\left(50 \mu \mathrm{mol} \mathrm{m} \mathrm{s}^{-1}\right.$ from above) for 21 days with or without having their roots shaded. Shoot lengths (A), root lengths (B), and nodule numbers (C) per plant were measured. Values are means \pm standard error (SE) (39 plants per treatment). The asterisk (*) indicates statistically significant at $P<0.05$ (Student's $t$ test). The experiment was carried out three times with similar results. D to E, Split-root plants were grown under continuous light $\left(50 \mu \mathrm{mol} \mathrm{m} \mathrm{m}^{-1}\right)$ for 21 days. Root lengths (D) and nodule numbers (E) were analyzed. Values are means \pm SE (18 plants per treatment). S/S indicates plants in which both root systems of the split root were shaded, whereas S/U refers to plants in which one side of the separated root system was shaded and the other was unshaded. Means denoted by the same letter do not differ significantly at $P<0.05$ (Tukey's multiple comparison test). The experiment was carried out three times with similar results. F, Nodulation in three distinct root zones. MG20 was grown under $16 \mathrm{~h} \mathrm{of} \mathrm{light}\left(150 \mu \mathrm{mol} \mathrm{m} \mathrm{m}^{-2} \mathrm{~s}^{-1}\right)$ and $8 \mathrm{~h}$ of dark from the side for 14 days. Roots were shaded in five different patterns: U, exposed to light (unshaded); S, dark (shaded with black plastic). Values are means \pm SE ( 24 plants per treatment). Means denoted by the same letter do not differ significantly at $P<0.05$ (Tukey's multiple comparison test). N.D. $=$ not detected. The experiment was carried out four times. 
that, although root lengths were not significantly different between the two different shaded (S/S) and shaded/unshaded $(\mathrm{S} / \mathrm{U})$ root systems (Fig. 1D), the overall number of nodules per root system was significantly reduced in the roots grown under completely unshaded conditions compared with those in shaded conditions (Fig. 1E). We used the data from Figure 1E and, as shown in Supplementary Figure S3A, prepared a graph combining the total number of nodules per split-root systems (S/S compared with the total number in the $\mathrm{S} / \mathrm{U}$ treatment). We next analyzed the expression of $\operatorname{nin}$, a nodulation gene marker, and found that its expression was significantly reduced on the unshaded root whereas nin was highly expressed in the shaded root in S/U plants, which were better nodulated. The reduction in $\operatorname{nin}$ expression was, thus, directly correlated with the reduced nodule number.

Finally, we investigated the effects of light on nodulation in a single root. The root of an inoculated plant growing in agar in a test tube was divided into three zones (proximal [top], middle, and distal [bottom]). Each zone was either exposed to light from the side (U, unshaded) or kept in the dark ( $\mathrm{S}$, shaded) by masking the test tube with black tape. First, we found that nodules developed only in fully shaded roots (SSS) and not in fully illuminated roots (UUU) (Fig. 1F), supporting the results shown in Figure 1C and E. The number of root nodules formed in the shaded zone was significantly higher than that in the unshaded zone of the USU roots. In SUU and UUS plants, nodulation was slightly but not significantly higher in the shaded zones than in the unshaded zones. In the roots with the midzone shaded (SSS or USU), nodulation was always higher than in the other SU treatments. Because the midzone of roots represents the most susceptible region for rhizobial infection and nodulation, this may explain the higher nodule number in this zone compared with other regions under shaded conditions. These data are in agreement with previous results showing that wild-type (WT) Medicago truncatula and L. japonicus develop root nodules in the susceptible zone (Nishimura et al. 2002a and b; Penmetsa and Cook 1997).

\section{Blue light inhibits nodulation.}

To determine which wavelength of light is critical for the inhibition of nodulation, we irradiated roots with blue or red light $\left(90 \mu \mathrm{mol} \mathrm{m} \mathrm{m}^{-2} \mathrm{~s}^{-1}\right)$ supplied from above for 21 days and analyzed nodulation thereafter. Although the light intensity in the soil at a depth of $10 \mathrm{~cm}$ would most likely be around $0.1 \mu \mathrm{mol} \mathrm{m} \mathrm{m}^{-2} \mathrm{~s}^{-1}$ under our treatment conditions, the unshaded and shaded roots were exposed to approximately $60 \mu \mathrm{mol} \mathrm{m} \mathrm{m}^{-2} \mathrm{~s}^{-1}$ and approximately $10 \mu \mathrm{mol} \mathrm{m} \mathrm{m}^{-2} \mathrm{~s}^{-1}$ of light, respectively.

Under red light, no significant differences in shoot and root length or in the number of root nodules between unshaded and shaded plants were observed (Fig. 2A to C). Under blue light, however, both shoot length and nodule number were significantly reduced in the unshaded plants and a slight decrease in root length was also observed (Fig. 2A to C). As for white light, we measured the shoot length of inoculated and uninoculated Lotus plants grown under blue light conditions to investigate whether the difference in shoot length under blue light with or without root shading was influenced by nodulation. As Supplementary Figure S4 illustrates, although no significant difference in shoot length between shaded and unshaded plants was seen for the uninoculated plants, the shoot lengths of shaded plants with inoculated roots were significantly increased, just as they were in white light. Thus, there is a strong correlation between shoot length and inoculation with rhizobia in both blue and white light. To investigate further whether the
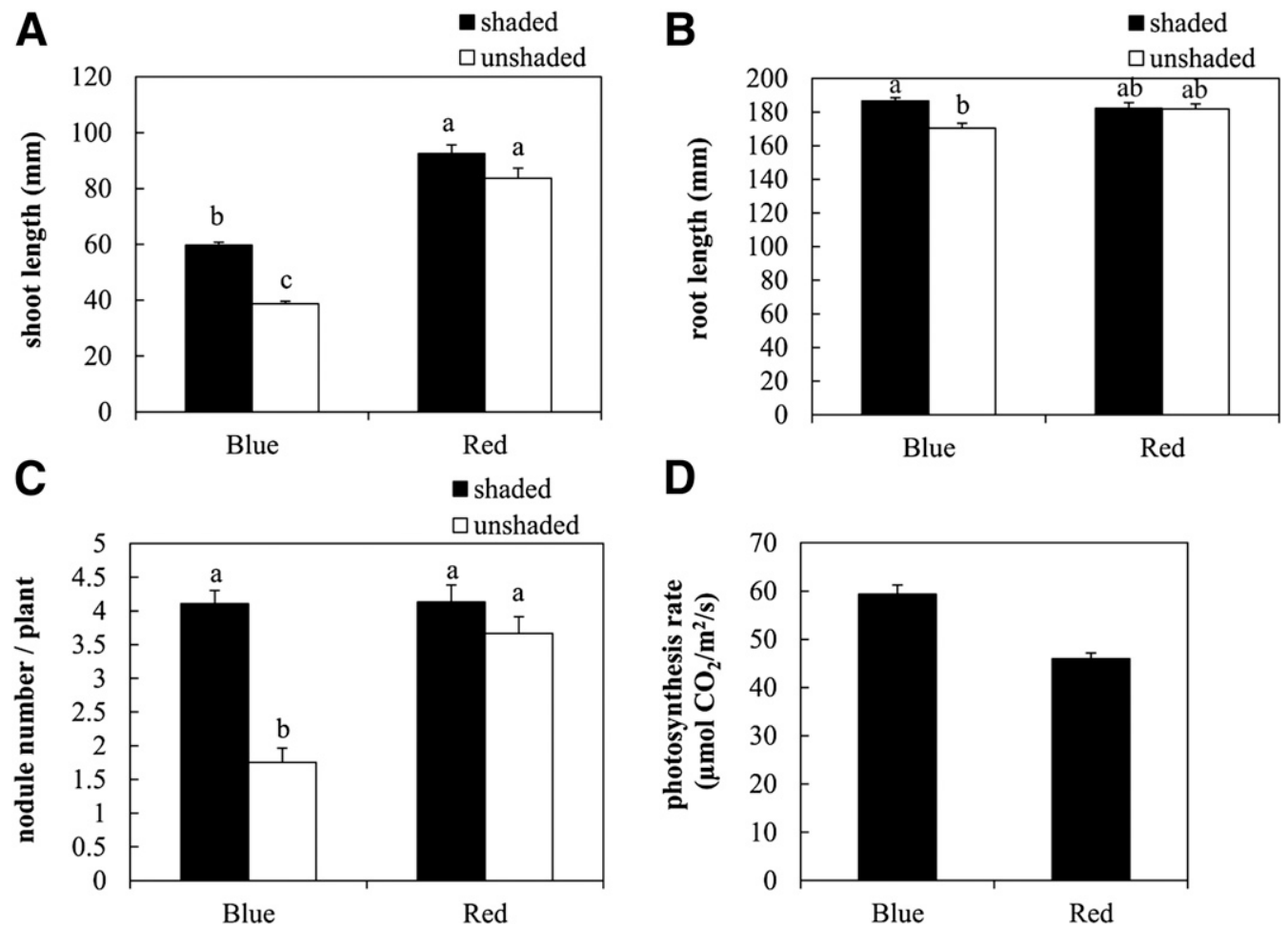

Fig. 2. Effects of blue and red light on nodulation. A to C, Inoculated MG20 was grown under blue or red light from above $\left(90 \mu \mathrm{mol} \mathrm{m} \mathrm{m}^{-2} \mathrm{~s}^{-1} ; 16 \mathrm{~h}\right.$ of light and $8 \mathrm{~h}$ of dark) for 21 days. Shoot lengths (A), root lengths (B), and the nodule numbers (C) per plant were measured. Values are means \pm standard error (SE) (85 plants per treatment). Means denoted by the same letter do not differ significantly at $P<0.05$ (Tukey's multiple comparison test). D, MG20 plants were irradiated with blue or red light $\left(90 \mu \mathrm{mol} \mathrm{m} \mathrm{s}^{-1}\right)$ for 21 days with the root shaded, and the photosynthesis rate was immediately measured. Five plants were combined into a single measurement. Values are means \pm SE (three replicates). No statistical significance was observed (Mann-Whitney $U$ test). All experiments were carried out three times. 
reduction in nodulation (Fig. 2C) in the illuminated roots was caused by lower photosynthetic activity under blue light, we measured the activity under both red and blue light and found that blue light did not lead to a reduced photosynthetic rate (Fig. 2D). This result strongly suggests that, in L. japonicus roots, nodulation is inhibited in response to blue light perception and not by reduced photosynthetic activity.

\section{Blue light inhibits rhizobial infection as well as nodule number.}

To analyze which step of the nodulation pathway is inhibited by blue light, both infection thread presence and nodule size class were measured. As shown in Figure 2C, not only did the number of nodules differ between the shaded and unshaded blue light-grown plants, but the number of infection threads per plant, as measured by DsRed fluorescence, was also reduced (Fig. 3A). The nodule size classes also differed between the two treatments. A lower proportion, i.e., 61 vs. $48 \%$ of the total number (193 shaded, 89 unshaded), were large $(>1.0 \mathrm{~mm})$ nodules and a greater number $(29 \%$ vs. $43 \%)$ were small $(0.5$ to $1.0 \mathrm{~mm})$ nodules in the unshaded plants compared with the shaded ones (Fig. 3B). Also, the fresh weight per nodule of the unshaded roots was significantly reduced compared with that of the shaded roots, reflecting nodule size (Fig. 3C). The same trend was observed in acetylene reduction activity (ARA) (Supplementary Fig. S5). Thus, in response to blue light, fewer infection threads were formed on the illuminated roots, causing a major decrease in nodule number and size. The ARA assay showed that the nodules that developed in unshaded roots were functional and that light did not directly affect nitrogen fixation but had an indirect effect by decreasing nodule number and weight.

\section{Reduced cryptochrome expression relieves inhibition of nodulation under blue light.}

The inhibition of nodulation by blue light suggested that the blue light photoreceptors - phototropins or cryptochromeswere involved. Using RNA interference (RNAi), we silenced the phototropin (phot1A, phot 1B, and phot 2 ) and cryptochrome (cry $1 A$, cry $1 B$, cry $2 A$, cry $2 B$, and cry3) genes in Agrobacterium rhizogenes-induced hairy roots of $L$. japonicus. First, the inhibition of target gene expression in induced hairy roots was confirmed by real-time reverse transcription-polymerase chain reaction (RT-PCR). In the RNAi plants, the expression of not only the target genes but, also, of closely related genes was suppressed (Fig. 4A to D; Supplementary Fig. S6A to C). For example, in roots carrying small interfering RNA (siRNA) for Ljphot1A, Ljphot1B, or Ljphot2, the levels of all Ljphot transcripts were lower than those in roots carrying the empty vector (EV). In LjcrylA-, Ljcry $1 B$-, Ljcry $2 A-$, and Ljcry $2 B$-targeted roots, the transcript levels of several Ljcry genes were lower than those in the EV control roots (Fig. 4A to D). Notably, in LjcrylA and $L j c r y 2 B$-silenced roots, all the other Ljcry transcripts (excluding Ljcry3) were reduced (Fig. 4A and D). In contrast, in Ljcry3-targeted roots, the expression of the other Ljcry genes was not affected nor was Ljcry3 expression decreased in any of the silenced roots (Fig. 4A to D). In Ljcry2B-RNAi plants, the exon sequence was similar to the sequences in LjcrylA, LjcrylB and Ljcry $2 A$ at, respectively, about 92,92 , and $90 \%(53,53$, and $152 \mathrm{bp}$ ), suggesting a possible cross-silencing effect. However, $\mathrm{Xu}$ et al. (2006) stated that at least 22-nt identity might be required for off-target silencing to occur. Because the $3^{\prime}$ untranslated region (UTR) was used for targeting the other Cry genes and because the similarity among them is low (not sufficiently high for siRNA) (Supplementary Fig. S7), the target sequence of these genes is likely to be gene specific. However, whether cross-silencing actually occurs is unknown (Supplementary Figs. S8 and S9).
When plants with hairy roots carrying an EV control were illuminated with blue light, root nodules formed on the shaded plants. Although nodulation was decreased more in the shaded hairy roots than in the blue light-illuminated control roots (Fig. $2 \mathrm{C}$ ), root nodulation was drastically suppressed on the unshaded hairy roots illuminated with blue light, as shown in Supplementary Figure S10. This result suggests that root nodulation is strongly inhibited by blue light in hairy roots. In contrast, root nodule numbers were significantly higher in cry $1 A$ - and cry $2 B$-targeted and unshaded roots and was correlated with a reduced expression of several Ljcry genes (except Ljcry3). No root nodules were observed in phot $1 B$ and
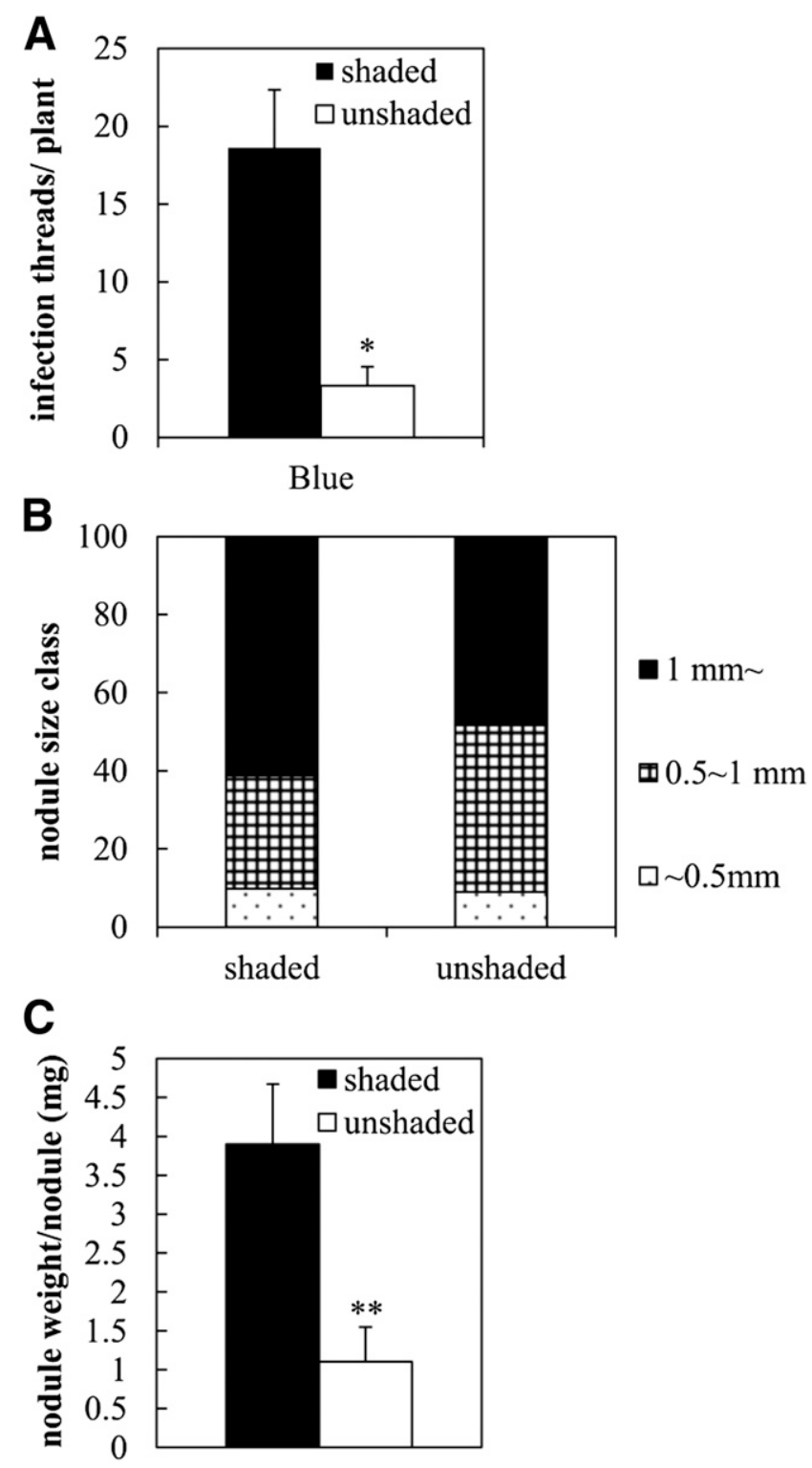

Fig. 3. Effect of light irradiation on the size of nodules formed and infection thread formation. A, Inoculated MG20 plants were grown under blue light from above $\left(90 \mu \mathrm{mol} \mathrm{m} \mathrm{m}^{-2} \mathrm{~s}^{-1} ; 16 \mathrm{~h}\right.$ of light and $8 \mathrm{~h}$ of dark) for 14 days, and infection threads were analyzed. Values are means \pm standard error (SE) (nine plants per treatment). An asterisk $(*)$ indicates statistically significant at $P<0.05$ (Mann-Whitney $U$ test). B, The nodule size class and $\mathbf{C}$, fresh weight per nodule of MG20 plants grown under blue light from above ( 90 $\mu \mathrm{mol} \mathrm{m} \mathrm{s}^{-2} \mathrm{~s}^{-1} ; 16 \mathrm{~h}$ of light and $8 \mathrm{~h}$ of dark) for 21 days were analyzed. The measured nodules numbered 193 (shaded roots) and 89 (unshaded roots) (B) and 67 (shaded roots) and 28 (unshaded roots) (C). Asterisks (**) indicate statistically significant at $P<0.01$ (Student's $t$ test). All experiments were carried out three times. 
phot2-silenced plants and reduced nodule numbers were measured in the phot1A RNAi plants, which is similar to the results for $c r y 1 B$ and $c r y 2 A$ plants (Table 1 ).

In addition, we investigated the expression of $\operatorname{nin}$, the nodulespecific gene, in EV and RNAi plants 21 days after $M$. loti inoculation. In EV and phot RNAi plants, no differences in nin transcript levels were observed, but in cry $1 A$ and $c r y 2 B$ RNAi plants, a trend toward higher nin expression was observed (Fig. $4 \mathrm{E}$ ). These results support a role for cryptochromes in blue light-induced inhibition of root nodulation.
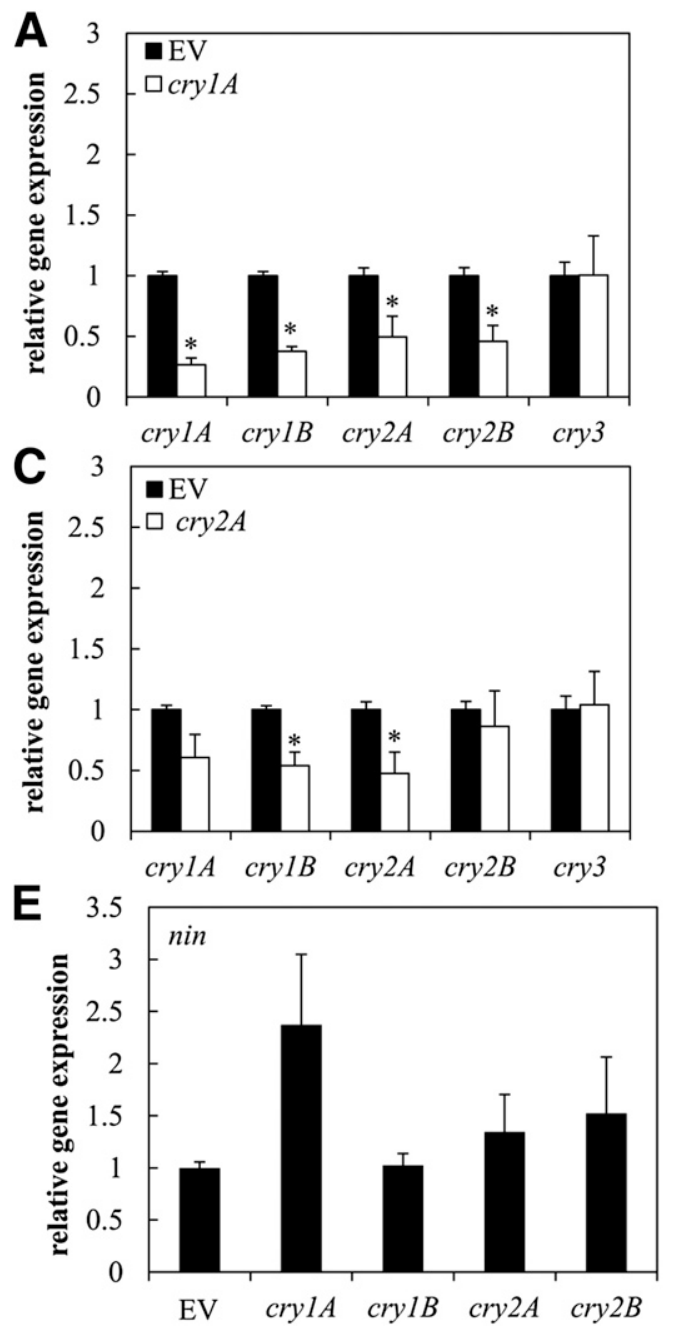

Rhizobial growth is inhibited by blue light.

To investigate whether light affects rhizobial growth, we exposed liquid cultures to blue or red light. Growth, measured by absorbance at $610 \mathrm{~nm}\left(A_{610}\right)$ of M. loti MAFF303099 under red light of either low or high intensity, was similar to that of the dark (Fig. 5A). On the other hand, low-intensity blue light significantly reduced $M$. loti growth and high-intensity blue light decreased it even more, as measured by absorbance, compared with the dark control (Fig. 5B). These results indicated that rhizobial growth is inhibited by blue light and that

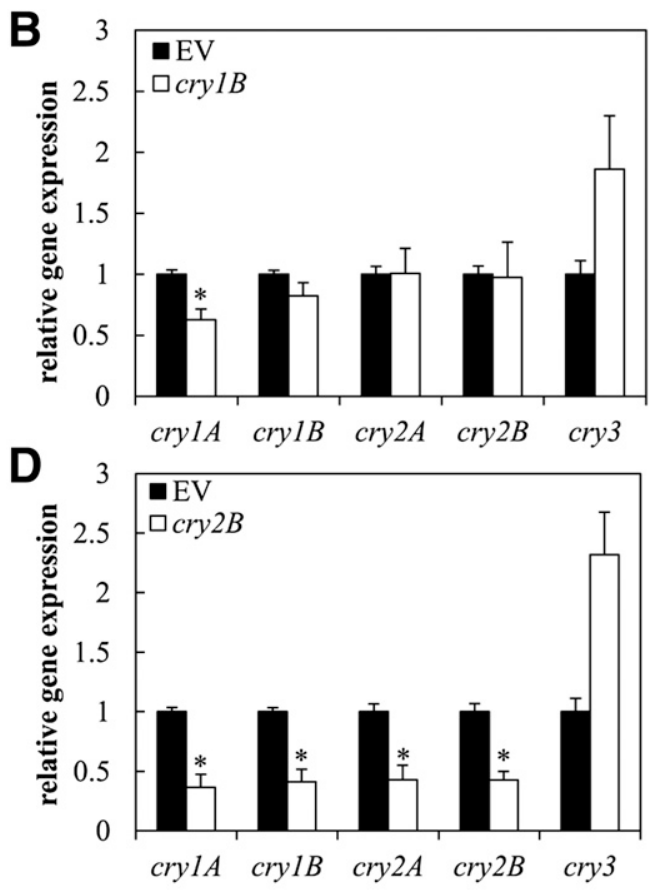

Fig. 4. Expression levels of target genes in RNA interference (RNAi) plants. A to D, Transcript levels of cryptochrome genes in empty vector (EV) and cryRNAi plants. E, Relative nin gene expression (normalized to that of ATP synthase as an internal control) in Mesorhizobium loti-inoculated EV and cry-RNAi plants. Values are means \pm standard error (SE) (nine plants per treatment). The significance of differences between EV and each $c r y$-RNAi plant was determined by the Mann-Whitney $U$ test; asterisks (*) indicate $P<0.05$.

Table 1. Nodulation in RNA interference (RNAi) plants under blue light (unshaded roots)

\begin{tabular}{|c|c|c|c|c|c|}
\hline \multirow[b]{2}{*}{ Target gene } & \multirow[b]{2}{*}{ No. of plants tested } & \multirow[b]{2}{*}{ No. of nodulated plants } & \multicolumn{2}{|c|}{ Average per plant } & \multirow[b]{2}{*}{ Significant difference (vs. empty vector) } \\
\hline & & & No. of nodules & Percentage & \\
\hline $\mathrm{EV}$ & 74 & 0 & 0 & $0 \%$ & - \\
\hline phot1A & 24 & 1 & 0.08 & $4 \%$ & n.s. \\
\hline phot1B & 17 & 0 & 0 & $0 \%$ & n.s. \\
\hline phot2 & 20 & 0 & 0 & $0 \%$ & n.s. \\
\hline crylA & 33 & 7 & 0.45 & $21 \%$ & $*$ \\
\hline cry $1 B$ & 29 & 2 & 0.14 & $7 \%$ & n.s. \\
\hline $\operatorname{cry} 2 A$ & 23 & 3 & 0.35 & $13 \%$ & n.s. \\
\hline $\operatorname{cry} 2 B$ & 23 & 5 & 0.19 & $22 \%$ & $*$ \\
\hline
\end{tabular}

${ }^{\mathrm{z}}$ Statistical significance is indicated by asterisks $(P<0.05$ by Student $t$ test $)$. n.s. indicates nonsignificant. 
the lack of rhizobial proliferation may be an explanation for the reduction in nodule number.

To investigate whether blue light has a direct effect on rhizobial growth, we transferred rhizobia that had been growing for $72 \mathrm{~h}$ in blue light to dark conditions and monitored their growth over the following $96 \mathrm{~h}$. After a lag period, rhizobial growth resumed in the dark at a rate similar to that of a culture maintained in the dark for the entire experiment (Fig. 5C). However, when the rhizobia were exposed to blue light for the entire time period, growth was negligible, whereas when cells grown in blue light were transferred to the dark at $72 \mathrm{~h}$, their $A_{610}$ values increased by $120 \mathrm{~h}$ after transer and the cells were growing at the same rate as the dark controls. These results strongly support the conclusion that rhizobial growth is inhibited specifically by blue light.

We then investigated the growth of two signature-tagged mutagenesis (STM) strains in M. loti (Shimoda et al. 2008) with disrupted genes homologous to LOV-HK/PAS protein ( $m l l p p 1$ ) and photolyase $(m l p h l)$. In the dark, the STM cell densities were similar to that of WT M. loti (Fig. 5D). However, high intensity blue light prevented the growth of WT M. loti, but after $72 \mathrm{~h}$, the growth of the STM strains was partially restored. The $A_{610}$ values of the WT and STM strains were significantly different at $144 \mathrm{~h}$ (Fig. 5E).

We also measured growth parameters in plants inoculated with the STM strains and exposed to blue light. For the shaded roots, no significant difference between the WT M. loti and STM strains was observed in shoot length (Fig. $5 \mathrm{~F}$ ), root length (Fig. 5G), or nodule number (Fig. 5H). For the unshaded plants, the nodule number of plants inoculated with WT M. loti was $40 \%$ of that of the shaded roots. For plants inoculated with the STM strains, the nodule count in unshaded roots was $>65 \%$ of the number for the shaded roots (Fig. 5H). Taken together, these results suggest that both sets of rhizobial blue light receptors are required to inhibit nodulation.
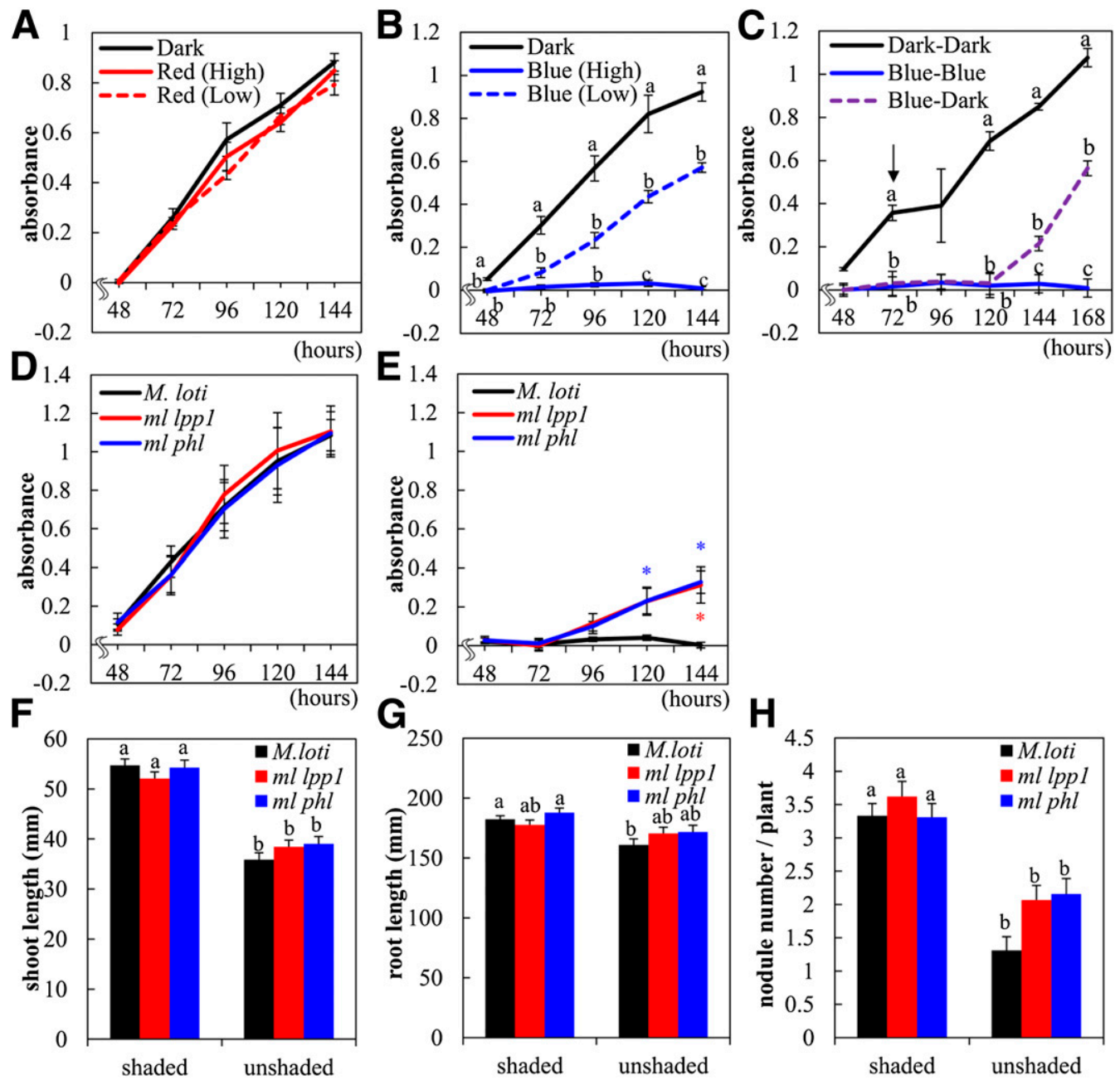

Fig. 5. Proliferation of Mesorhizobium loti MAFF303099 and signature-tagged mutagenesis (STM) strains and nodulation of Lotus japonicus. A, Wild-type M. loti (MAFF303099) was cultured under red or B, blue light at a high $\left(90 \mu \mathrm{mol} \mathrm{m}^{-2} \mathrm{~s}^{-1}\right)$ or low intensity $\left(25 \mu \mathrm{mol} \mathrm{m}{ }^{-2} \mathrm{~s}^{-1}\right)$. Values are means \pm standard error (SE) (12 samples [A] and 10 samples [B]). Means denoted by the same letter do not differ significantly at $P<0.05$ (Tukey-Kramer multiple comparison test). The experiment was carried out four times with similar results. C, Wild-type $M$. loti was cultured in the dark or under blue light $\left(90 \mu \mathrm{mol} \mathrm{m} \mathrm{m}^{-2} \mathrm{~s}^{-1}\right)$ for $72 \mathrm{~h}$ (arrow). Dark-grown M. loti was kept in the dark until $168 \mathrm{~h}$ (Dark-Dark); blue light-grown M. loti cells either remained under the same conditions until $168 \mathrm{~h}$ (Blue-Blue) or were moved to the dark (Blue-Dark). Values are means \pm SE (four samples per treatment). Means denoted by the same letter do not differ significantly at $P<0.05$ (Tukey's multiple comparison test). The experiment was carried out two times with similar results. D, Growth of STM strains and wildtype (WT) $M$. loti in the dark or $\mathbf{E}$, under high-density blue light was analyzed. Values are means \pm SE (two samples per treatment). Asterisks (*) indicate statistically significant at $P<0.05$ (Mann-Whitney $U$ test) in comparison with WT $M$. loti. The experiment was carried out two times with similar results. F, Shoot length, G, root length, and $\mathbf{H}$, the nodule number per plant of MG20 plants inoculated with WT $M$. loti or the STM strains, shaded or unshaded and grown under blue light from above $\left(90 \mu \mathrm{mol} \mathrm{m}{ }^{-2} \mathrm{~s}^{-1} ; 16 \mathrm{~h}\right.$ of light and $8 \mathrm{~h}$ of dark). Values are means $\pm \mathrm{SE}$ ( 45 plants per treatment). Means denoted by the same letter do not differ significantly at $P<0.05$ (Tukey's multiple comparison test). The experiment was carried out three times with similar results. 


\section{Nodulation is increased additively}

\section{in RNAi plants inoculated with STM strains.}

Because blue light perception by both the root and rhizobia leads to an inhibition of nodulation, we inoculated RNAi plants with the STM mutants under the same growth conditions used in Table 1 and assessed nodulation status 21 dai. As shown in Table 2, root nodules were formed in cry-RNAi plants with M. loti or in EV plants inoculated with STM strains, supporting the results shown in Table 1 and Figure $5 \mathrm{H}$. The nodulation of RNAi-targeted Ljcry $1 A$ and Ljcry $2 B$ plants when inoculated by mllppl or mlphl was increased compared with EV plants inoculated with mllppl or mlphl. In RNAi-targeted LjcrylA and Ljcry $2 B$ plants inoculated with the mllppl strain or the mlphl strain, nodulation was enhanced compared with EV plants inoculated with a WT $M$. loti strain (Table 2). The increase in nodule number was additive, indicating that the inhibition of nodulation by light is caused by blue light perception by both the host plant roots and rhizobia.

\section{DISCUSSION}

Previous studies showed that nodulation in Trifolium subterraneum L. Woogenellup is either inhibited or not inhibited in the light, depending on the rhizobial strain (Gibson 1968). On the other hand, in Pisum sativum (Rudin 1956) and P. vulgaris (Grobbelaar et al. 1971), root nodule numbers were decreased when roots were exposed to light. Our data support both of these seemingly disparate conclusions because, in L. japonicus, inhibition of nodulation by light is caused by blue light perception by both the host plant roots and rhizobia. Bonomi et al. (2012) reported that a short time exposure of rhizobia to white light prior to inoculation enhanced nodulation in Pisum sativum. In our study, a light treatment was given after inoculating with rhizobia, which may explain the differences in results.

Using a split-root system, we found that the number of nodules formed on unshaded root systems was significantly lower compared with shaded root systems and even on a single root, which showed that nodule inhibition was not systemic (Fig. 1E and F).

Recently, Chen et al. (2014) reported that the shoot lengths of Oryza sativa seedlings were inhibited by blue light and, in Lactuca sativa, shoot biomass was also decreased under blue light (Fujiuchi and Fujiwara 2012). Furthermore, shoot and hypocotyl lengths of lettuce, radish, and pepper decreased in response to increasing the quantity of both blue light and red light (Cope et al. 2014). Although the number of nodules did not differ between the red and blue treatments, the shoot lengths of blue light-treated plants in shaded L. japonicus roots were significantly decreased compared with red light-treated plants (Fig. 2A).

In lettuce, root growth was decreased by blue light irradiation (Fujiuchi and Fujiwara 2012). In contrast, in rice, root growth was not different between roots either exposed to blue light or not (Chen et al. 2014). Our observation of root growth was that no large difference occurred whether the roots were shaded or unshaded in L. japonicus (Fig. 2B). Taken together, these results show that overall shoot growth is inhibited by blue light irradiation, whereas the effect of blue light irradiation on root growth depends on the plant species.

Under both white and blue light, a significant difference was seen in the shoot lengths of inoculated plants that had their roots shaded compared with uninoculated plants grown under the same conditions. These data lead us to conclude that the shoot growth of shaded roots was positively affected by the presence of root nodules.

We also found that the inhibition of nodulation in white light (Fig. 1) was caused by its blue component (Fig. 2) and that root nodule number reduction under blue light was not related to the lack of a carbon source (Fig. 2D). Similarly, for isolated $P$. vulgaris roots, blue light inhibited nodulation more than red light or white light did (Grobbelaar et al. 1971). Our data are thus consistent with the absence of a significant effect of red light on root nodule number in Pisum sativum (Lie 1969) and, also, show that number of root nodules following blue light treatment was significantly reduced (Fig. 2C).

Further implicating the key role of blue light, we found that nodulation was significantly increased in roots depleted of $c r y 1 A$ and $\operatorname{cry} 2 B$ (Table 1). Because the expression of all the Ljcryl and Ljcry 2 genes was down-regulated in both $c r y 1 A$ and $c r y 2 B$ RNAi plants, we conclude that blue light inhibits nodulation via one or both cryptochromes 1 and 2. On the other hand, reduced expression of the three phototropin genes in L. japonicus MG20 had no effect on nodulation, indicating that blue light perception affects nodule development in the roots of this legume through cryptochromes and not phototropins. We also demonstrated that $M$. loti growth is inhibited specifically by blue light and that the rhizobial blue light receptors LPP1 and photolyase are involved in its suppression (Fig. 5E). Moreover, the growth of the STM strains under blue light (Fig. 5E) did not lead to a full recovery of the levels of the dark-grown strains (Fig. 5D), which means that both rhizobial photoreceptors are involved.

Finally, when the Ljcry $1 A$ and Ljcry $2 B$-targeted RNAi plants were inoculated with the STM strains, an additive increase in nodule number was observed (Table 2). These results thus demonstrate that the inhibition of nodulation by light is caused by blue light perception by both host plant roots and rhizobia.

With regard to nodulation, the number of infection threads per plant in L. japonicus is severely reduced in plants grown under blue light (Fig. 3A). Nodule size classes also differed between the shaded and unshaded plants. In the latter, a large population of smaller-sized nodules and only a few large ones developed on the illuminated roots compared with the shaded controls (Fig. 3B), suggesting that the inhibition of L. japonicus MG20 root nodulation under blue light results from one or both

Table 2. Nodulation of RNA interference (RNAi) plants with signature-tagged mutagenesis strains under blue light (unshaded roots)

\begin{tabular}{|c|c|c|c|c|c|}
\hline Inoculated rhizobia & Target gene ${ }^{y}$ & No. of plants tested & No. of nodulated plants & Percentage & Average per plant ${ }^{\mathrm{z}}$ \\
\hline \multirow{3}{*}{ Mesorhizobium loti } & EV & 74 & 0 & $0 \%$ & $0 \mathrm{a}$ \\
\hline & crylA & 63 & 9 & $14 \%$ & $0.27 \mathrm{a}$ \\
\hline & $\operatorname{cry} 2 B$ & 61 & 15 & $25 \%$ & $0.48 \mathrm{ab}$ \\
\hline \multirow[t]{3}{*}{ mllpp1 } & EV & 24 & 6 & $25 \%$ & $0.38 \mathrm{ab}$ \\
\hline & CrylA & 34 & 11 & $32 \%$ & $0.68 \mathrm{abc}$ \\
\hline & cry $2 B$ & 36 & 21 & $58 \%$ & $1.56 \mathrm{bc}$ \\
\hline \multirow[t]{3}{*}{ mlphl } & EV & 27 & 4 & $15 \%$ & $0.30 \mathrm{a}$ \\
\hline & crylA & 35 & 11 & $31 \%$ & $0.89 a b c$ \\
\hline & $\operatorname{cry} 2 B$ & 39 & 20 & $51 \%$ & $1.90 \mathrm{c}$ \\
\hline
\end{tabular}

\footnotetext{
${ }^{\mathrm{y}} \mathrm{EV}=$ empty vector.

${ }^{\mathrm{z}}$ Average number of nodules per plant. Different letters are significantly different (Tukey-Kramer multiple comparison test, $P<0.05$ ).
} 
reduced or abnormal infection. Nodule fresh weights are also lower in the illuminated plants.

At least two hypotheses can be invoked to explain these results. The first is based on the fact that light perception reduces rhizobial growth, resulting in insufficient size of the rhizobial population needed for inducing the earliest stages of nodule formation. This scenario is consistent with the studies on Rhizobium leguminosarum bv. viciae 3841 in which white light was reported to interfere with the synthesis of bacterial surface determinants that are needed for competent infection and nodulation of pea (Bonomi et al. 2012). Inhibition of attachment to root surfaces might also result in fewer infection events with a concomitant reduction in nodule size and weight, which we observed.

An alternative or additional mechanism is based on the observations of Grobbelaar et al. (1971) that light perception affects nodule development independently of the early stages of attachment, root hair deformation, and infection thread formation, which would mean that darkness is not required for nodule initiation but for nodule organogenesis. Our data are also consistent with this mechanism because we observed a larger size class of nodules in the 0.5 - to $1-\mathrm{mm}$ range and a reduction in the group of 1-mm-sized nodules in the shaded vs. unshaded roots. In addition, the fresh weight per nodule under shaded conditions was greater than when the roots were illuminated. However, the fact that fewer mature nodules were observed on unshaded roots could solely be due to the abortion or lack of the initial nodulation attempts under blue light. We are currently examining which of these two mechanisms is more likely to explain the downstream effects of blue light in the M. loti-L. japonicus symbiosis.

Higher plants developed avoidance mechanisms to survive under conditions of biotic or abiotic stress. It is well-recognized that plants utilize light as the trigger for these responses. For example, in the case of anthogenesis and light-induced germination, the plant determines the timing by light perception through phytochromes and cryptochromes to increase the possibility of survival of descendants (Blázquez and Weigel 1999; Devlin and Kay 2000; Shi et al. 2015). In root negative phototropism, plant roots bend opposite to the light direction via phototropins (Haga et al. 2005; Kanegae et al. 2000). In the shade avoidance syndrome, plants grow taller or bend toward the light to avoid shade through the action of phytochromes (Smith and Whitelam 1997). In shade avoidance in the case of root nodulation and mycorrhization, host plants suppress these interactions under suboptimal light conditions through the phytochrome system to avoid expending energy (Nagata et al. 2015; Suzuki et al. 2011). Therefore, we can consider inhibition of nodulation by light as one of several avoidance responses plants use to conserve energy in response to environmental stress. Sun et al. (2005) reported that the light from the surrounding environment of herbaceous plants enters the interior of the stem. Then, via an internal light-conducting system, light of wavelengths between 710 and $940 \mathrm{~nm}$, which includes far-red (FR) light, is conducted axially and efficiently from leaves and stems toward underground roots, whereas lower wavelengths of light (400 to $710 \mathrm{~nm}$ ), such as blue, are not. These authors also stated that this internal light environment might be of crucial importance for the phytochrome-regulated metabolic activities of plant stems and roots. Because the phytochrome system is involved in the response to FR light conducted to the root, another system, such as blue light perception, may be utilized for avoidance mechanisms in response to stress. Clearly, more studies are needed.

\section{MATERIALS AND METHODS}

\section{Plant material and genes.}

L. japonicus Miyakojima MG20 was the source of the plant material. The sequences of the genes identified and reported in this paper have been deposited in the L. japonicus genome database (Miyakogusa.jp ver. 3.0 database). Phototropin genes were designated phot $1 A(\mathrm{Lj} 3 \mathrm{~g} 3 \mathrm{v} 3117660.5)$, phot $1 B(\mathrm{Lj} 0 \mathrm{~g} 3 \mathrm{v} 0322849.1)$, and phot 2 (Lj0g3v0090469.2); cryptochrome genes were designated crylA (Lj0g3v0286829.3), crylB (Lj0g3v0011469.1), cry2A (Lj6g3v0029980.2), cry2B (Lj5g3v1203330.2), and cry3 (Lj1g3v1965120.1). Identities of nucleotide sequences between the above sequences and closest matches from Arabidopsis thaliana were as follows: Ljphot1A (81\%) and Ljphot1B (77\%) vs. Atphot1 (AF360218); Ljphot2 (82\%) vs. Atphot2 (AF053941); LjcrylA (78\%) and Ljcry1B (81\%) vs. Atcryl (AY124863); Ljcry2A (81\%) and Ljcry2B (79\%) vs. Atcry2 (BT008576); and Ljcry3 (89\%) vs. Atcry3 (AY102138) (Supplementary Fig. S11).

\section{Rhizobial strains.}

Mesorhizobium loti MAFF303099 (Kaneko et al. 2000; Keele et al. 1969; Saeki and Kouchi 2000) and four STM M. loti strains with disrupted photoreceptor-related genes were used for inoculation. At the beginning of this study, we planned to identify genes homologous to phototropin and cryptochrome. For phototropin homologs, an M. loti sequence with an amino acid sequence similarity of the ml19655 about $49.2 \%$ (295 aa in length to pRL110320; the LOV/PAS proteins of Rhizobium leguminosarum [Bonomi et al. 2012]) was detected and designated as MILPP. For the cryptochrome homologs, the similarity of the mll8094 amino acid sequence to all Ljcry family members is approximately $40 \%$ and to that of blr5310 (Bradyrhizobium japonicum) 50.8\% (443 aa in length, annotated as photolyase). Thus, we designated this gene as Mlphotolyase. The gene sequences reported in this paper have been deposited in the Rhizobium genome database RhizoBase. In the STM strains, transposon insertions, e.g., plasposon plasmid pTnModOGm, which carries a Tn5-based mini-transposon, randomly distribute throughout the genome (Shimoda et al. 2008). In this mutagenesis strategy, one STM strain has one mutation. The STM strains were obtained from the National Bio Resource Project (NBRP) and, in this study, STM strains mll9655 (clone ID: 38T01b03, 09T04f12, and 17T03h11) and mll8094 (clone ID: 06T03e06) were designated mllpp1, mllpp2, mllpp3, and $m l p h l$, respectively. Although mllpp has three alleles, these alleles have a similar phenotype (Supplementary Fig. S12). Therefore, we used mllpp1, in which the mutation had been inserted near the 5' UTR.

\section{Nodulation tests and size measurement of nodules.}

Seeds were germinated on $0.8 \%$ (wt/vol) agar for 3 days in the dark. The seedlings (with roots shaded with black paper outside of the plate) were grown on $1.5 \%$ (wt/vol) agarsolidified $\mathrm{N}$-free Broughton and Dilworth (B\&D) medium (Broughton and Dilworth 1971) for 7 days under white light. Roots were then inoculated with M. loti MAFF303099 or STM strains $\left(1.0 \times 10^{7}\right.$ cells per plant $)$, and the plants were grown for 21 days under various light conditions in a vertical orientation. A fluorescent lamp was used for white light $\left(50 \mu \mathrm{mol} \mathrm{m} \mathrm{m}^{-2} \mathrm{~s}^{-1}\right)$ and light-emitting diodes (LH-80LED-DT made by Nippon Medical \& Chemical Instruments Co., Ltd., Osaka, Japan) (50\% bandwidth: $20 \mathrm{~nm})$ for blue $(448 \mathrm{~nm})$ or red $(660 \mathrm{~nm})$ light $\left(90 \mu \mathrm{mol} \mathrm{m} \mathrm{m}^{-2} \mathrm{~s}^{-1}\right)$. Light was supplied from above.

The fresh weight of detached nodules was obtained, and the size classes of the nodules were measured under the dissecting microscope 21 dai.

\section{ARA assay for nitrogenase activity.}

Seeds were germinated on $0.8 \%$ (wt/vol) agar for 3 days in the dark. The seedlings (with roots shaded with black paper outside of the plate) were grown on $1.5 \%$ (wt/vol) agar-solidified 
$\mathrm{N}$-free B\&D medium for 7 days under white light. Roots were then inoculated with $M$. loti $\left(1.0 \times 10^{7}\right.$ cells per plant $)$, and the plants were grown for 21 days under blue light conditions in a vertical orientation. Five plants were placed in each 34-ml test tube, which was then covered with a rubber serum cap and was degassed for $30 \mathrm{~s}$. Acetylene that was diluted five times was injected into the tube, which was then incubated in a $25^{\circ} \mathrm{C}$ chamber. After $1.5 \mathrm{~h}$ of incubation, the amount of ethylene was determined by gas chromatography.

\section{Observation of infection threads.}

MG20 seeds were sown on $0.8 \%$ (wt/vol) agar medium and were incubated at $24^{\circ} \mathrm{C}$ in the dark. After 3 days, germinated seedlings were transplanted onto $1.5 \%$ agar-solidified $\mathrm{B} \& \mathrm{D}$ medium without nitrogen and were incubated under white light. The root areas of the square plates were shaded using black paper. After 7 days, the plants were inoculated with DsRed (Maekawa et al. 2009) labeled M. loti and moved to blue light conditions with or without the roots being shaded. Infection threads were observed 14 days later under a fluorescent microscope (SZX9 Olympus) and were counted.

\section{Split-root system.}

A split-root system was prepared, using adventitious roots. Seeds were germinated on $0.8 \%$ (wt/vol) agar for 1 day in the dark, followed by 1 day in $16 \mathrm{~h}$ of white light and $8 \mathrm{~h}$ of dark. The seedling roots were removed, leaving a root fragment approximately $1 \mathrm{~mm}$ long, and the seedlings were then grown on $1.0 \%(\mathrm{wt} / \mathrm{vol})$ agar-solidified $\mathrm{N}$-free $\mathrm{B} \& \mathrm{D}$ medium with naphthalene acetic acid $(0.5 \mu \mathrm{g} / \mathrm{ml})$ for 3 days. They were later transplanted to $1.0 \%$ (wt/vol) agar-solidified N-free B\&D medium containing aminoethoxyvinylglycine $(1 \mu \mathrm{M})$ and were grown for 14 days with the extending roots shaded by black paper attached outside of the plate. Following removal of the paper and separation of the root system into two parts, the plants were inoculated with M. loti and were grown for 21 days under the same light and dark cycle with or without root shading of the root sections. To shade the roots, a $0.5-\mathrm{mm}$-thick piece of black plastic was placed directly on the root inside of the plate and the outside of the plate was masked with black tape. The unshaded roots were covered with clear plastic inside of the plate to replicate any potential physical stress that may have occurred on the shaded side.

\section{Nodulation test in a single root.}

Three-day-old seedlings were transplanted into test tubes (22 $\mathrm{mm} \times 240 \mathrm{~mm}$ ) filled to a depth of $180 \mathrm{~mm}$ with B\&D agar medium (one seedling per tube). Seedlings were grown for 14 days (until the root reached the bottom of the tube) and were then inoculated with $M$. loti. The proximal, middle, and distal parts of each root were either masked with black tape outside the test tube ( $\mathrm{S}$, shaded) or exposed to the light (U, unshaded). Five masking patterns (SSS, SUU, USU, UUS, and UUU) were used. The plants were then grown under white light $(150 \mu \mathrm{mol}$ $\mathrm{m}^{-2} \mathrm{~s}^{-1}, 16 \mathrm{~h}$ of light and $8 \mathrm{~h}$ of dark) for 14 days, and the root nodules were counted.

\section{Photosynthesis rate analyses.}

Plants were irradiated with blue or red light $\left(90 \mu \mathrm{mol} \mathrm{m}^{-2} \mathrm{~s}^{-1}\right)$ for 21 days with their roots shaded, and the photosynthesis rate was measured with a miniPPM-300 plant photosynthesis meter (EARS, Delft, The Netherlands). Five plants were used for a single measurement.

\section{Generation of RNAi transformants.}

A PCR-amplified DNA fragment of the 3' UTR region or exon region $(c r y 2 B)$ of each target gene was cloned into the
pENTR/D-TOPO entry vector (Invitrogen, Carlsbad, CA, U.S.A.) The inserts of the entry clones were recloned into the pUBGWS-GFP vector (Maekawa et al. 2008) (obtained from NBRP) by LR reaction, using the Gateway cloning system (Invitrogen). The resultant plasmid or EV (negative control) was introduced into Agrobacterium rhizogenes LBA1334 as follows. Seeds were germinated for 4 days in the dark. Seedling hypocotyls were cut off, the cut surface was infected with $A$. rhizogenes, and shoots without roots were cocultured for 5 days in the dark. To induce hairy root formation, cocultured plants were grown for 14 days on $1.5 \%$ B5 agar medium (Gamborg et al. 1968) (16 h of light and $8 \mathrm{~h}$ of dark). Plants with green fluorescent protein positive hairy roots were selected under a fluorescence microscope (SZX9, Olympus, Tokyo). Selected transformants were inoculated with rhizobia $\left(1.0 \times 10^{7}\right.$ cells $)$, were grown on agar-solidified $\mathrm{N}$-free B\&D medium for 21 days, and were irradiated with blue light $\left(90 \mu \mathrm{mol} \mathrm{m} \mathrm{m}^{-2} \mathrm{~s}^{-1}\right)$ without root shading.

\section{Real-time RT-PCR.}

Plant roots were quickly frozen in liquid $\mathrm{N}_{2} 21$ dai and were stored at $-80^{\circ} \mathrm{C}$. Total RNA was prepared with an RNeasy plant mini kit (Qiagen, Basel, Switzerland). DNase I treatment was performed with DNase RT-Grade (Wako, Osaka, Japan). A onestep SYBR Primescript RT-PCR kit (Takara, Shiga, Japan) was used. Transcript levels were normalized against the LjATP synthase transcript. The nucleotide sequences of the primers used are shown in Supplementary Table S1.

\section{Rhizobial growth.}

Rhizobia $\left(1.0 \times 10^{7}\right.$ cells $\left.\mathrm{ml}^{-1}\right)$ were diluted $1: 10$ with yeats mannitol liquid medium and were cultured without shaking at $25^{\circ} \mathrm{C}$, under red or blue light at high intensity $\left(90 \mu \mathrm{mol} \mathrm{m}^{-2} \mathrm{~s}^{-1}\right)$ or low intensity $\left(25 \mu \mathrm{mol} \mathrm{m} \mathrm{m}^{-2} \mathrm{~s}^{-1}, 16 \mathrm{~h}\right.$ of light and $8 \mathrm{~h}$ of dark). To estimate bacterial growth, the absorbance $\left(A_{610}\right)$ of the bacterial culture was measured every $24 \mathrm{~h}$ from 48 to 144 or $168 \mathrm{~h}$.

\section{ACKNOWLEDGMENTS}

Lotus japonicus Miyakojima MG20 seeds were provided by the National BioResource Project of the Ministry of Education, Culture, Sports, Science and Technology, Japan. We thank T. Maekawa for the preliminary results on blue light-dependent bacterial growth.

\section{LITERATURE CITED}

Ahmad, M., and Cashmore, A. R. 1993. HY4 gene of A. thaliana encodes a protein with characteristics of a blue light photoreceptor. Nature 366: $162-166$.

Auldridge, M. E., and Forest, K. T. 2011. Bacterial phytochromes: More than meets the light. Crit. Rev. Biochem. Mol. Biol. 46:67-88.

Bethlenfalvay, G. J., and Phillips, D. A. 1977. Effect of light intensity on efficiency of carbon dioxide and nitrogen reduction in Pisum sativum L. Plant Physiol. 60:868-871.

Blázquez, M. A., and Weigel, D. 1999. Independent regulation of flowering by phytochrome B and gibberellins in Arabidopsis. Plant Physiol. 120: 1025-1032.

Bonomi, H. R., Posadas, D. M., Paris, G., Carrica, Mdel. C., Frederickson, M., Pietrasanta, L. I., Bogomolni, R. A., Zorreguieta, A., and Goldbaum, F. A. 2012. Light regulates attachment, exopolysaccharide production, and nodulation in Rhizobium leguminosarum through a LOVhistidine kinase photoreceptor. Proc. Natl. Acad. Sci. U.S.A. 109: 12135-12140.

Briggs, W. R., Beck, C. F., Cashmore, A. R., Christie, J. M., Hughes, J., Jarillo, J. A., Kagawa, T., Kanegae, H., Liscum, E., Nagatani, A., Okada, K., Salomon, M., Rüdiger, W., Sakai, T., Takano, M., Wada, M., and Watson, J. C. 2001. The phototropin family of photoreceptors. Plant Cell 13:993-997.

Broughton, W. J., and Dilworth, M. J. 1971. Control of leghaemoglobin synthesis in snake beans. Biochem. J. 125:1075-1080. 
Chen, C.-C., Huang, M.-Y., Lin, K.-H., Wong, S.-L., Huang, W.-D., and Yang, C.-M. 2014. Effects of light quality on the growth, development and metabolism of rice seedlings (Oryza sativa L.). J. Biotechnol. 9: 13-24.

Christie, J. M., Blackwood, L., Petersen, J., and Sullivan, S. 2015. Plant flavoprotein photoreceptors. Plant Cell Physiol. 56:401-413.

Christie, J. M., Reymond, P., Powell, G. K., Bernasconi, P., Raibekas, A. A., Liscum, E., and Briggs, W. R. 1998. Arabidopsis NPH1: A flavoprotein with the properties of a photoreceptor for phototropism. Science 282: 1698-1701.

Christie, J. M., Salomon, M., Nozue, K., Wada, M., and Briggs, W. R. 1999. LOV (light, oxygen, or voltage) domains of the blue light photoreceptor phototropin (nph1): Binding sites for the chromophore flavin mononucleotide. Proc. Natl. Acad. Sci. U.S.A. 96:8779-8783.

Cope, K. R., Snowden, M. C., and Bugbee, B. 2014. Photobiological interactions of blue light and photosynthetic photon flux: Effects of monochromatic and broad-spectrum light sources. Photochem. Photobiol. 90:574-584.

Devlin, P. F., and Kay, S. A. 2000. Cryptochromes are required for phytochrome signaling to the circadian clock but not for rhythmicity. Plant Cell 12:2499-2510.

Fujiuchi, N., and Fujiwara, K. 2012. LED dim light irradiation of the root zone influences growth and development of leaf lettuce (Lactuca sativa) plants under nutrient film technique hydroponics. Envrion. Control Biol. 50:101-106

Gamborg, O. L., Miller, R. A., and Ojima, K. 1968. Nutrient requirements of suspension cultures of soybean root cells. Exp. Cell Res. 50:151158.

Gibson, A. H. 1968. Nodulation failure in Trifolium subterraneum L. cv. Woogenellup (Syn. Marrar). Aust. J. Agric. Res. 19:907-918.

Giraud, E., Fardoux, J., Fourrier, N., Hannibal, L., Genty, B., Bouyer, P., Dreyfus, B., and Verméglio, A. 2002. Bacteriophytochrome controls photosystem synthesis in anoxygenic bacteria. Nature 417:202205.

Gressel, J. 1979. Blue light photoreception. Photochem. Photobiol. 30: 749-754.

Grobbelaar, N., Clarke, B., and Hough, M. C. 1971. The nodulation and nitrogen fixation of isolated roots of Phaseolus vulgaris L. II. The influence of light on nodulation. Plant Soil 35:203-214.

Haga, K., Takano, M., Neumann, R., and Iino, M. 2005. The rice COLEOPTILE PHOTOTROPISM1 gene encoding an ortholog of Arabidopsis $\mathrm{NPH}_{3}$ is required for phototropism of coleoptiles and lateral translocation of auxin. Plant Cell 17:103-115.

Han, I.-S., Cho, H.-Y., Moni, A., Lee, A.-Y., and Briggs, W. R. 2013. Investigations on the photoregulation of chloroplast movement and leaf positioning in Arabidopsis. Plant Cell Physiol. 54:48-56.

Huala, E., Oeller, P. W., Liscum, E., Han, I. S., Larsen, E., and Briggs, W. R. 1997. Arabidopsis NPH1: A protein kinase with a putative redox-sensing domain. Science 278:2120-2123.

Kanegae, H., Tahir, M., Savazzini, F., Yamamoto, K., Yano, M., Sasaki, T. Kanegae, T., Wada, M., and Takano, M. 2000. Rice NPH1 homologues, $O s N P H 1 a$ and $O s N P H 1 b$, are differently photoregulated. Plant Cell Physiol. 41:415-423.

Kaneko, T., Nakamura, Y., Sato, S., Asamizu, E., Kato, T., Sasamoto, S., Watanabe, A., Idesawa, K., Ishikawa, A., Kawashima, K., Kimura, T., Kishida, Y., Kiyokawa, C., Kohara, M., Matsumoto, M., Matsuno, A., Mochizuki, Y., Nakayama, S., Nakazaki, N., Shimpo, S., Sugimoto, M., Takeuchi, C., Yamada, M., and Tabata, S. 2000. Complete genome structure of the nitrogen-fixing symbiotic bacterium Mesorhizobium loti. DNA Res. 7:331-338

Keele, B. B., Jr., Hamilton, P. B., and Elkan, G. H. 1969. Glucose catabolism in Rhizobium japonicum. J. Bacteriol. 97:1184-1191.

Li, Y.-Y., Mao, K., Zhao, C., Zhang, R.-F., Zhao, X.-Y., Zhang, H.-L., Shu, H.-R., and Zhao, Y. J. 2013. Molecular cloning of cryptochrome 1 from apple and its functional characterization in Arabidopsis. Plant Physiol. Biochem. 67:169-177.

Lie, T. A. 1969. Non-photosynthetic effects of red and far-red light on rootnodule formation by leguminous plants. Plant Soil 30:391-404.

Maekawa, T., Kusakabe, M., Shimoda, Y., Sato, S., Tabata, S., Murooka, Y., and Hayashi, M. 2008. Polyubiquitin promoter-based binary vectors for overexpression and gene silencing in Lotus japonicus. Mol. PlantMicrobe Interact. 21:375-382.

Maekawa, T., Maekawa-Yoshikawa, M., Takeda, N., Imaizumi-Anraku, H., Murooka, Y., and Hayashi, M. 2009. Gibberellin controls the nodulation signaling pathway in Lotus japonicus. Plant J. 58:183-194.

Mockler, T., Yang, H., Yu, X., Parikh, D., Cheng, Y.-C., Dolan, S., and Lin, C. 2003. Regulation of photoperiodic flowering by Arabidopsis photoreceptors. Proc. Natl. Acad. Sci. U.S.A. 100:2140-2145.
Nagata, M., Yamamoto, N., Shigeyama, T., Terasawa, Y., Anai, T., Sakai, T., Inada, S., Arima, S., Hashiguchi, M., Akashi, R., Nakayama, H., Ueno, D., Hirsch, A. M., and Suzuki, A. 2015. Red/far red light controls arbuscular mycorrhizal colonization via jasmonic acid and strigolactone signaling. Plant Cell Physiol. 56:2100-2109.

Nishimura, R., Ohmori, M., Fujita, H., and Kawaguchi, M. 2002b. A Lotus basic leucine zipper protein with a RING-finger motif negatively regulates the developmental program of nodulation. Proc. Natl. Acad. Sci. U.S.A. 99:15206-15210.

Nishimura, R., Ohmori, M., and Kawaguchi, M. 2002a. The novel symbiotic phenotype of enhanced-nodulating mutant of Lotus japonicus: astray mutant is an early nodulating mutant with wider nodulation zone. Plant Cell Physiol. 43:853-859.

Ogura, Y., Komatsu, A., Zikihara, K., Nanjo, T., Tokutomi, S., Wada, M., and Kiyosue, T. 2008a. Blue light diminishes interaction of PAS/LOV proteins, putative blue light receptors in Arabidopsis thaliana, with their interacting partners. J. Plant Res. 121:97-105.

Ogura, Y., Tokutomi, S., Wada, M., and Kiyosue, T. 2008b. PAS/LOV proteins: A proposed new class of plant blue light receptor. Plant Signal. Behav. 3:966-968.

Oh, E., Kang, H., Yamaguchi, S., Park, J., Lee, D., Kamiya, Y., and Choi, G. 2009. Genome-wide analysis of genes targeted by PHYTOCHROME INTERACTING FACTOR 3-LIKE5 during seed germination in Arabidopsis. Plant Cell 21:403-419.

Penmetsa, R. V., and Cook, D. R. 1997. A legume ethylene-insensitive mutant hyperinfected by its rhizobial symbiont. Science 275:527530.

Purcell, E. B., and Crosson, S. 2008. Photoregulation in prokaryotes. Curr. Opin. Microbiol. 11:168-178.

Rudin, P. E. 1956. Versuche zur Physiologie der Knöllchenbildung bei Pisum sativum L. Phytopathol. Z. 28:57-80.

Saeki, K., and Kouchi, H. 2000. The lotus symbiont, Mesorhizobium loti: Molecular genetic techniques and application. J. Plant Res. 113: 457-465.

Sakai, T., and Haga, K. 2012. Molecular genetic analysis of phototropism in Arabidopsis. Plant Cell Physiol. 53:1517-1534.

Sasaki, T., Suzaki, T., Soyano, T., Kojima, M., Sakakibara, H., and Kawaguchi, M. 2014. Shoot-derived cytokinins systemically regulate root nodulation. Nat. Commun. 5:4983.

Sharma, P., Chatterjee, M., Burman, N., and Khurana, J. P. 2014. Cryptochrome 1 regulates growth and development in Brassica through alteration in the expression of genes involved in light, phytohormone and stress signalling. Plant Cell Environ. 37:961-977.

Shi, H., Wang, X., Mo, X., Tang, C., Zhong, S., and Deng, X. W. 2015. Arabidopsis DET1 degrades HFR1 but stabilizes PIF1 to precisely regulate seed germination. Proc. Natl. Acad. Sci. U.S.A. 112:38173822 .

Shimoda, Y., Mitsui, H., Kamimatsuse, H., Minamisawa, K., Nishiyama, E., Ohtsubo, Y., Nagata, Y., Tsuda, M., Shinpo, S., Watanabe, A., Kohara, M., Yamada, M., Nakamura, Y., Tabata, S. and Sato, S. 2008. Construction of signature-tagged mutant library in Mesorhizobium loti as a powerful tool for functional genomics. DNA Res. 15:297-308.

Smith, H., and Whitelam, G. C. 1997. The shade avoidance syndrome: Multiple responses mediated by multiple phytochromes. Plant Cell Environ. 20:840-844

Sun, J., Cardoza, V., Mitchell, D. M., Bright, L., Oldroyd, G., and Harris, J. M. 2006. Crosstalk between jasmonic acid, ethylene and Nod factor signaling allows integration of diverse inputs for regulation of nodulation. Plant J. 46:961-970.

Sun, Q., Yoda, K., and Suzuki, H. 2005. Internal axial light conduction in the stems and roots of herbaceous plants. J. Exp. Bot. 56:191-203.

Suzuki, A., Suriyagoda, L., Shigeyama, T., Tominaga, A., Sasaki, M., Hiratsuka, Y., Yoshinaga, A., Arima, S., Agarie, S., Sakai, T., Inada, S., Jikumaru, Y., Kamiya, Y., Uchiumi, T., Abe, M., Hashiguchi, M. Akashi, R., Sato, S., Kaneko, T., Tabata, S., and Hirsch, A. M. 2011 Lotus japonicus nodulation is photomorphogenetically controlled by sensing the red/far red (R/FR) ratio through jasmonic acid (JA) signaling. Proc. Natl. Acad. Sci. U.S.A. 108:16837-16842.

Thompson, C. L., and Sancar, A. 2002. Photolyase/cryptochrome blue light photoreceptors use photon energy to repair DNA and reset the circadian clock. Oncogene 21:9043-9056.

van der Horst, M. A., Key, J., and Hellingwerf, K. J. 2007. Photosensing in chemotrophic, non-phototrophic bacteria: Let there be light sensing too Trends Microbiol. 15:554-562.

Weller, J. L., Foo, E. M., Hecht, V., Ridge, S., Vander Schoor, J. K., and Reid, J. B. 2015. Ethylene signaling influences light-regulated development in pea. Plant Physiol. 169:115-124. 
Xu, P., Zhang, Y., Kang, L., Roossinck, M. J., and Mysore, K. S. 2006. Computational estimation and experimental verification of off-target silencing during posttranscriptional gene silencing in plants. Plant Physiol. 142:429-440.

Zhao, X., Li, Y.-Y., Xiao, H. L., Xu, C.-S., and Zhang, X. 2013. Nitric oxide blocks blue light-induced $\mathrm{K}^{+}$influx by elevating the cytosolic $\mathrm{Ca}^{2+}$ concentration in Vicia faba L. guard cells. J. Integr. Plant Biol. 55:527-536. Zuanazzi, J. A. S., Clergeot, P. H., Quirion, J.-C., Husson, H.-P., Kondorosi, A., and Ratet, P. 1998. Production of Sinorhizobium meliloti nod gene activator and repressor flavonoids from Medicago sativa roots. Mol. Plant-Microbe Interact. 11:784-794.

\section{AUTHOR-RECOMMENDED INTERNET RESOURCES}

Miyakogusa.jp ver. 3.0 database: http://www.kazusa.or.jp/lotus National Bio Resource Project LegumeBase database: http://www.legumebase.brc.miyazaki-u.ac.jp

RhizoBase database: http://genome.microbedb.jp/RhizoBase 